\title{
Conocimiento enfermero en la farmacología del TDAH
}

\author{
José Miguel Fontestad Gómez \\ al365399@uji.es \\ Victoria Gil Felip \\ al362163@uji.es \\ Elena Cátedra García \\ al362126@uji.es \\ Sara Heredia Nebot \\ al362125@uji.es \\ María Vicenta Lucas Miralles \\ mlucas@uji.es
}

\section{Resumen}

Introducción: El trastorno por déficit de atención e hiperactividad (TDAH) es un trastorno que afecta a un $5 \%$ de los adolescentes y entre un 2,5-4\% de los adultos, teniendo un gran impacto social. El objetivo principal de esta revisión es mostrar cuáles son los principales tratamientos para el TDAH, priorizando un orden de elección.

Metodología: Se realizó una revisión de la literatura, escogiendo ensayos clínicos y revisiones sistemáticas sobre el tratamiento farmacológico del TDAH y los efectos secundarios asociados. Para ello, se realizó la búsqueda empleando los Descriptores en Ciencias de la Salud (DeCS) y los Medical Subject Headings (MeSH) en las siguientes bases de datos: PubMed, Cochrane y Scopus.

Resultados: Se incluyeron 10 estudios que realizan una evaluación económica completa y de seguridad sobre el metilfenidato (MPH), las anfetaminas, entre ellas principalmente la lisdexanfetamina (LDX), y la atomoxetina (ATX).

Conclusión: El tratamiento idóneo sería el MPH, puesto que cuenta con más ensayos sobre su seguridad y efectividad. Sin dejar de contemplar la LDX, que ha demostrado ser ligeramente superior al MPH en relación costeefectividad; pero, al ser más nueva, no cuenta con la misma evidencia que el $\mathrm{MPH}$. Además, sería importante, tal y como reflejan los autores, un control exhaustivo de los tratados con estas sustancias que padezcan de patologías cardiovasculares, sobre todo en sus efectos a largo plazo, donde no encontramos suficiente literatura. 
Palabras clave: trastorno por déficit de atención e hiperactividad, tratamiento farmacológico, fármacos estimulantes del sistema nervioso central, efectos adversos.

\section{Abstract}

Introduction: Attention deficit hyperactivity disorder (ADHD) is a disorder that affects $5 \%$ of adolescents and $2.5-4 \%$ of adults, having a big social impact. The primary objective is to know the main treatments for ADHD, prioritizing an order of choice.

Methodology: An integrative review was carried out, choosing: clinical trials and systematic reviews about ADHD, drug treatments and its adverse effects. The literature search was done with Medical Subject Headings and Health Science Descriptors in different databases (PubMed, Cochrane and Scopus).

Results: We include 10 studies conducting a comprehensive and safety assessment of methylphenidate (MPH), amphetamines, including mainly the lisdexamfetamine (LDX), and atomoxetine (ATX).

Conclusion: The ideal treatment would be MPH, as it has more tests on its safety and effectiveness. While contemplating LDX, which has been shown to be slightly higher than MPH in cost-effectiveness; but, as it is more recent, it does not have the same evidence as the MPH. In addition, exhaustive control of those treated with these substances and suffering from cardiovascular pathologies would be important, especially in its long-term effects where we do not find enough literature.

Keywords: attention deficit hyperactivity disorder, drug therapy, central nervous system stimulants, adverse effects.

\section{Introducción}

El trastorno por déficit de atención e hiperactividad (TDAH) es la principal condición neuropsiquiátrica del desarrollo tanto en niños como en adolescentes: se estima que un $5 \%$ del total de este grupo lo padece, reduciéndose la prevalencia al llegar a la edad adulta entre un 2,5-4 \% en todo el mundo. Se observa una ligera disminución con la edad; además, muchos jóvenes suelen dejar de precisar tratamiento con el paso del tiempo (Vásquez et al. 2010).

Cuando se habla de la fisiopatología del TDAH, existen diversas teorías, pero la más apoyada es el retraso en la maduración cerebral, en concreto, del cerebro ejecutivo que se localiza en la corteza prefrontal. Este es responsable de medir las consecuencias de los actos o las respuestas de los demás, causando su inmadurez una incapacidad para inhibir en el afectado sus respuestas, para organizarse y controlar la atención o la conducta, además de la incapacidad de hacer frente a las demandas que se le presentan (Alda-Díez et al. 2017).

Para realizar el diagnóstico diferencial, se tendrán en cuenta ciertos puntos según el manual diagnóstico DSM-V, entre estos se encuentran los principales síntomas del trastorno que han de persistir durante un periodo mínimo de 6 meses y que, como mínimo, uno debe estar presente antes de los 12 años (Vásquez et al. 2010): 
- Inatención: por lo general, no son capaces de mantener la atención en los estudios, en ver una película o cualquier otra cosa (Soutollo-Esperón y Díez-Suárez 2007).

- Hiperactividad: actividad excesiva e inapropiada tanto para la edad como para la situación o la tarea (Soutollo-Esperón y Díez-Suárez 2007).

- Impulsividad: no valoran interiormente las diferentes posibilidades y consecuencias de sus actos (Soutollo-Esperón y Díez Suárez 2007).

Respecto a estos síntomas, al llegar a la edad adulta, la hiperactividad se atenúa o transforma en un nerviosismo interno, por lo que se estereotipa el TDAH como un trastorno infanto-juvenil. Mientras, la impulsividad y la inatención se mantienen sin cambios.

EI TDAH es una condición de etiología multifactorial en la que intervienen principalmente factores genéticos y ambientales, además de neuroquímicos, neuroanatómicos, fisiológicos y psicosociales.

Como trastorno del cerebro ejecutivo, acarrea una serie de problemas en aquellas personas afectadas, como lo son las dificultades sociales, psicológicas y de aprendizaje; así, existen otras complicaciones asociadas: el fracaso académico, el consumo de sustancias ilegales, una alta tasa de delincuencia y la aparición de problemas comórbidos psiquiátricos como la depresión o la ansiedad principalmente (Vásquez et al. 2010).

Tras diversos ensayos se demostró una inhibición de ciertos neurotransmisores en la corteza prefrontal de los afectados por TDAH. De este modo, el tratamiento está enfocado en aumentar la concentración de los neurotransmisores inhibidos (dopamina y noradrenalina) en dicha área. Para lograr este objetivo, se emplean dos grupos de fármacos: los estimulantes y los no estimulantes (Soutollo-Esperón y Díez Suárez 2007).

Previo a la revisión de los tratamientos farmacológicos actuales para controlar los síntomas del TDAH, es de interés conocer el camino recorrido hasta la actualidad. Antiguamente los fármacos estimulantes de elección fueron las anfetaminas, en concreto una mezcla de cuatro sales; más recientemente, a finales de siglo, se optó por la dextroanfetamina, el metilfenidato y la pemolina de magnesio. Respecto a los no estimulantes se pautaban los antidepresivos tricíclicos, los $\alpha_{2}$-agonistas (clonidina) y los neurolépticos (clorpromazina) (Boerlin, Gitlin, Zoellner y Hammen 1998).

Al gran impacto clínico y social de este trastorno se le debe sumar la elevada carga económica empleada en el tratamiento: estos jóvenes precisan de atención médica especializada continuada y tratamientos farmacológicos diarios que ascienden la cuantía monetaria a la cifra de 9860-14 $483 €$ en Europa por paciente al año. A esta cifra se le debe sumar que algunos niños necesitan un consumo mayor de recursos al precisar un abordaje más amplio del problema, apareciendo en este punto la psicoterapia (Karlstad et al. 2016).

De este modo, como objetivo general del presente trabajo se establece: identificar los principales tratamientos farmacológicos del TDAH con el fin de contar con profesionales sanitarios que tengan una formación sobre los mismos, para lograr un ahorro económico y obtener una mejora en el bienestar del niño y de su entorno más cercano. Un niño diagnosticado de TDAH podrá ser tratado desde pequeño; además, sus padres y profesores serán asesorados a la hora de educar al menor, por lo que se 
podrán evitar situaciones adversas que en el futuro repercutirán tanto en el niño como en su familia. Asimismo, los objetivos específicos son:

- Identificar cuál es el medicamento de primera elección en el tratamiento del TDAH.

- Mencionar otros tipos de fármacos que pueden ser utilizados para tratar los síntomas del TDAH.

- Detallar los posibles efectos adversos que produce la medicación en estos pacientes.

\section{Metodología}

En cuanto al diseño de este estudio, se ha realizado revisión de la literatura y se han escogido ensayos clínicos y revisiones sistemáticas sobre el tratamiento farmacológico del TDAH y los efectos secundarios asociados.

En el siguiente trabajo se han empleado una serie de recursos para la obtención de la mejor evidencia disponible. Estos corresponden a una serie de bases de datos que cuentan con revisiones y artículos relacionados con las ciencias de la salud. Se han establecido diferentes DeCS y MeSH que se han combinado con la ayuda del operador booleano AND para realizar las búsquedas en las bases de datos: PubMed, Cochrane Library y SCOPUS.

En PubMed se realizaron las dos búsquedas siguientes:

- Central Nervous System Stimulants (Pharmacological Action) AND Atomoxetine Hydrochloride AND Attention Deficit Disorder with Hyperactivity.

- Lisdexamfetamine Dimesylate AND Attention Deficit Disorder with Hyperactivity .

En Scopus se empleó una sola fórmula:

- Lisdexamfetamine Dimesylate AND Attention Deficit Disorder with Hyperactivity.

Finalmente, en Cochrane Library se hizo también una única búsqueda:

- Attention Deficit Disorder with Hyperactivity AND Drug Therapy.

Una vez finalizada la búsqueda, indagamos cuáles concordaban con los objetivos decididos previamente; para ello, empleamos los criterios de inclusión y exclusión de nuestro trabajo: artículos publicados en un periodo no superior a los últimos cinco años, abiertos al público y basados en investigaciones realizadas en humanos. Toda esta información se encuentra en las tablas 1 y 2 . 
Tabla 1

Descriptores utilizados (fuente: elaboración propia)

\begin{tabular}{|c|c|c|c|}
\hline Lenguaje natural & $\begin{array}{l}\text { DeCs } \\
\text { (castellano) }\end{array}$ & Decs (inglés) & Mesh \\
\hline $\begin{array}{l}\text { Trastorno por déficit } \\
\text { de atención e } \\
\text { hiperactividad }\end{array}$ & $\begin{array}{l}\text { Trastorno por } \\
\text { déficit de atención } \\
\text { con hiperactividad }\end{array}$ & $\begin{array}{lr}\text { Attention } & \text { deficit } \\
\text { disorder } & \text { with } \\
\text { hyperactivity } & \end{array}$ & $\begin{array}{l}\text { Attention } \\
\text { disorder } \\
\text { hyperactivity }\end{array}$ \\
\hline Metilfenidato & Metilfenidato & Methylphenidate & Methylphenidate \\
\hline Lisdexanfetamina & $\begin{array}{l}\text { Dimesilato de } \\
\text { lisdexanfetamina }\end{array}$ & $\begin{array}{l}\text { Lisdexamfetamine } \\
\text { Dimesylate }\end{array}$ & $\begin{array}{l}\text { Lisdexamfetamine } \\
\text { Dimesylate }\end{array}$ \\
\hline Atomoxetina & $\begin{array}{l}\text { Clorhidrato } \\
\text { atomoxetina }\end{array}$ & $\begin{array}{l}\text { Atomoxetine } \\
\text { Hydrochloride }\end{array}$ & $\begin{array}{l}\text { Atomoxetine } \\
\text { Hydrochloride }\end{array}$ \\
\hline $\begin{array}{l}\text { Tratamiento } \\
\text { farmacológico }\end{array}$ & $\begin{array}{l}\text { Tratamiento } \\
\text { farmacológico }\end{array}$ & Drug Therapy & Drug Therapy \\
\hline $\begin{array}{l}\text { Fármacos } \\
\text { estimulantes del } \\
\text { sistema nervioso } \\
\text { central }\end{array}$ & $\begin{array}{l}\text { Estimulantes del } \\
\text { sistema nervioso } \\
\text { central }\end{array}$ & $\begin{array}{l}\text { Central Nervous } \\
\text { System Stimulants }\end{array}$ & $\begin{array}{l}\text { Central Nervous } \\
\text { System Stimulants } \\
\text { (Pharmacological } \\
\text { action) }\end{array}$ \\
\hline
\end{tabular}

Tabla 2

Estrategia de búsqueda por base de datos (fuente: elaboración propia)

\begin{tabular}{llll}
\hline $\begin{array}{l}\text { Bases } \\
\text { datos }\end{array}$ & de & Estrategia de búsqueda & Resultados \\
\hline PubMed & $\begin{array}{l}\text { Central Nervous System Stimulants } \\
\text { AND Atomoxetine Hydrochloride AND Attention Deficit Disorder } \\
\text { with Hyperactivity }\end{array}$ & 32 \\
\cline { 2 - 4 } & $\begin{array}{l}\text { Lisdexamfetamine Dimesylate AND Attention Deficit Disorder } \\
\text { with Hyperactivity }\end{array}$ & 206 \\
\hline Scopus & $\begin{array}{l}\text { Lisdexamfetamine Dimesylate AND Attention Deficit Disorder } \\
\text { with Hyperactivity }\end{array}$ & 209 \\
\hline $\begin{array}{l}\text { Cochrane } \\
\text { Library }\end{array}$ & Attention Deficit Disorder with Hyperactivity AND Drug Therapy & 26 \\
\hline
\end{tabular}

Por último, se ha empleado un manual de diagnóstico y tratamiento del TDAH, de la editorial Médica Panamericana, y una guía clínica para el manejo del TDAH, del Instituto Nacional de Psiquiatría Ramón de la Fuente. 


\section{Resultados}

Al realizar la búsqueda bibliográfica en las tres bases de datos seleccionadas, como podemos apreciar en la tabla 2, sin aplicar ningún tipo de limitador se obtuvieron 109 artículos. Aplicando los limitadores correspondientes en cada una de las bases de datos seleccionadas, el número total de artículos se redujo a 99, tal y como se observa en la tabla 3.

Tabla 3

Número de artículos encontrados en la búsqueda (fuente: elaboración propia)

\begin{tabular}{|c|c|c|}
\hline $\begin{array}{l}\text { Bases de } \\
\text { datos }\end{array}$ & Artículos encontrados sin limitadores & $\begin{array}{l}\text { Artículos recuperados con la } \\
\text { aplicación de limitadores }\end{array}$ \\
\hline \multirow[t]{2}{*}{ PubMed } & $\begin{array}{l}\text { Central Nervous System Stimulants } \\
\text { (Pharmacological Action) AND Atomoxetine } \\
\text { Hydrochloride AND Attention Deficit Disorder } \\
\text { with Hyperactivity }\end{array}$ & $\begin{array}{l}\text { Limitadores: Free full text; } \\
\text { published in the last } 5 \text { years; } \\
\text { Articled basades on humans. } \\
n=54\end{array}$ \\
\hline & $\begin{array}{l}\text { Lisdexamfetamine Dimesylate AND Attention } \\
\text { Deficit Disorder with Hyperactivity } \\
\mathrm{n}=60\end{array}$ & \\
\hline Scopus & $\begin{array}{l}\text { Lisdexamfetamine Dimesylate AND Attention } \\
\text { Deficit Disorder with Hyperactivity } \\
\mathrm{n}=30\end{array}$ & $\begin{array}{l}\text { Limitadores: Texto completo; } \\
\text { Fecha de publicación 2014-2019; } \\
\text { Artículos basados en humanos. } \\
\mathrm{n}=28\end{array}$ \\
\hline $\begin{array}{l}\text { Cochrane } \\
\text { Library }\end{array}$ & $\begin{array}{l}\text { Attention Deficit Disorder with Hyperactivity AND } \\
\text { Drug Therapy } \\
n=19\end{array}$ & $\begin{array}{l}\text { Limitadores: Texto completo; } \\
\text { Fecha de publicación 2014-2019; } \\
\text { Artículos basados en humanos. } \\
n=17\end{array}$ \\
\hline TOTAL & 109 & 99 \\
\hline
\end{tabular}

En relación a las búsquedas sobre el metilfenidato, destacamos los artículos que tratan sobre la lisdexanfetamina (LDX) y atomoxetina (ATX), de los cuales pudimos trabajar con 54 artículos publicados en la base de datos de PubMed y 28 en Scopus únicamente de LDX.

De los 99 estudios obtenidos en las bases de datos, no todos fueron seleccionados; los motivos fueron: no encontrarse disponible de forma abierta para el público, no tener relación con el tema a tratar y encontrarse artículos repetidos. El número final de artículos con los que proseguir la revisión y a los que realizar la lectura reflexiva fue de 10.

Estos 10 artículos sobre los que hemos trabajado constan de 5 revisiones sistemáticas, 3 ensayos clínicos, 1 estudio de cohortes y 1 revisión bibliográfica, siendo todos ellos artículos publicados recientemente con un máximo de antigüedad del año 2014. 


\section{Discusión y conclusión}

De acuerdo con la literatura basada en la evidencia, hay dos grandes bloques de psicofármacos para el tratamiento del TDAH: en primer lugar, los estimulantes, donde encontramos como gran referente al metilfenidato (MPH) y las anfetaminas (AMPH) entre cuyos derivados destaca la lisdexanfetamina (LDX), la cual está demostrando su eficacia en recientes estudios. En segundo lugar, tenemos los no estimulantes, donde destacaría la atomoxetina (ATX).

Con respecto a lo anterior, nos encontramos con la opinión de diversos autores (Newcorn et al. 2017), los cuales realizaron una revisión sistemática de estudios donde se demuestra que el MPH y la LDX son significativamente similares a la hora de reducir los síntomas del TDAH, sugiriendo que ambos fármacos pueden emplearse como primera línea de tratamiento en niños y adolescentes. En su revisión también se comenta que el cambio entre MPH y LDX se puede realizar sin ningún problema o efecto adverso cuando alguno de los tratamientos no produzca una respuesta positiva en el paciente tratado.

En la misma línea encontramos el estudio de Zimovetz, Joseph, Ayyagari y Mauskopf (2018) quienes realizaron otra revisión sistemática. En esta revisión se compara, en adultos con TDAH, el MPH con la LDX, la ATX y los placebos, llegando a la conclusión de que ninguno tiene efectos secundarios significativos. A pesar de eso, se comenta que, según el índice coste/efectividad, el fármaco por excelencia es la LDX, seguida por el MPH y, en último lugar, la ATX.

Centrándonos en el MPH, Storebø et al. (2015) realizaron otra revisión sistemática. Según la revisión, el MPH mejora los principales síntomas del TDAH y la calidad de vida de los niños y adolescentes afectados. Secundariamente a esto destaca que el MPH está asociado a efectos secundarios leves como insomnio e hiporexia, sin incrementar otros efectos graves como la muerte súbita. Asimismo, los autores afirman que se debería investigar más en los efectos adversos del MPH.

Por su parte, Punja et al. (2016) realizaron una revisión sistemática sobre las AMPH y sus derivados. En esta revisión afirman que estos fármacos reducen los síntomas tempranos del TDAH, aunque están asociados con un número mayor de efectos adversos, sin diferenciar el uso de AMPH de vida media larga y el uso de las de vida media corta. Es necesario investigar más este grupo de estimulantes.

Siguiendo con la comparación entre fármacos, Zimovetz et al. (2016) realizaron un ensayo clínico para valorar cuál sería la mejor opción (entre ATX y LDX) en caso de que el MPH fallase como primera opción en el tratamiento de niños y adolescentes con TDAH. El ensayo contó con dos grupos de comparación: el primero tratado con ATX y el segundo, con LDX. El ensayo se realizó en dos periodos: el primero, un periodo de valoración de 28 días para comprobar si había una correcta respuesta inicial al tratamiento con LDX y ATX, y el segundo periodo de posvaloración, que duró 48 semanas, para valorar cuál de los dos tratamientos era más eficiente en cuanto a efectividad y coste. Una vez acabado el periodo, se llegó a la conclusión de que la LDX controlaba de forma más rápida y superior los síntomas del TDAH en aquellos pacientes que respondieron mal al MPH. Como conclusión, según su modelo de coste efectividad y como defienden también otros autores (Dittmann et al. 2013), encontraríamos en primer lugar al MPH, en segundo lugar, la LDX y, en tercer lugar, la ATX. En contraposición a este ensayo, King et al. (2006) afirman en su revisión sistemática que se debe elegir siempre como primera opción el $\mathrm{MPH}$, como segunda la dexanfetamina y en último lugar la ATX, sin llegar a contemplar la LDX. 
Pasando a valorar los efectos secundarios de estos fármacos, encontramos a Berg et al. (2014), quienes realizaron un ensayo donde afirman que los fármacos (en general) para el tratamiento del TDAH han demostrado un aumento de la frecuencia cardiaca y de la presión arterial. Esto es poco relevante para niños sanos, pero en niños con patología cardiaca supondría un problema, así que recomiendan realizar un examen físico con las pruebas necesarias antes de iniciar el tratamiento, además de hacer un seguimiento activo por parte del profesional, puesto que se desconocen los efectos adversos que estos fármacos pueden provocar en los niños más vulnerables.

Siguiendo con los efectos adversos de estos fármacos, en la revisión bibliográfica de Hennissen et al. (2017) se nombran los principales efectos secundarios que son el insomnio y la hiporexia. Se comparan también los efectos cardiovasculares que puedan producir el MPH, la LDX y la ATX. Los tres fármacos son simpaticomiméticos, por lo que tienen efectos sobre el sistema cardiovascular, alterando de este modo la presión arterial diastólica, la sistólica y la frecuencia cardiaca principalmente. A pesar de estos problemas, se siguió con el consumo de estos fármacos, ya que se observó que se resolvían espontáneamente o cambiando la posología. El estudio llegó a la conclusión de que, a corto plazo, los efectos cardiovasculares son escasos sin haber apenas diferencias en los tres fármacos, aunque el MPH es el que menos valores altera de los tres. Por otro lado, incide en que no hay suficientes estudios que demuestren su seguridad cardiaca a largo plazo, puesto que en un futuro podrían surgir problemas como la hipertrofia ventricular y las retinopatías, entre otros, por lo que se recomienda activamente un seguimiento por parte del profesional sanitario a los niños y adolescentes tratados.

Por último, como se ha explicado en la introducción, los pacientes con TDAH suelen padecer problemas comórbidos, entre los que cabe destacar el fracaso escolar y la asociada depresión. En este aspecto destacan Linden et al. (2016), quienes realizaron un estudio de cohortes para comprobar si los fármacos utilizados en el tratamiento aumentaban el riesgo suicida. Este estudio comparaba la atomoxetina con los estimulantes y concluía que ambos son fármacos seguros a corto plazo, justificando que, si había una pequeña tasa mayor de suicidios en la ATX comparada con los estimulantes, era porque la ATX es un fármaco de segunda línea que se instaura en personas más mayores y con más desafíos por delante.

Como conclusión, tras llevar a cabo el análisis crítico de la literatura basado en la evidencia y comparar los diferentes resultados obtenidos sobre el tratamiento del TDAH, hemos observado que el MPH es la primera opción de tratamiento ya que es el fármaco que presenta más estudios de investigación a su favor. Cabe destacar el LDX, considerado también como tratamiento de primera opción por su rápida acción y disminución de los síntomas, con el que se obtienen además mejores resultados que con el MPH en adolescentes y adultos, según el índice coste-efectividad. Por otro lado, el tratamiento con AMPH necesita más estudios de investigación ya que reduce los síntomas a corto plazo, pero se ha contemplado una gran cantidad de efectos adversos. La ATX sería el fármaco de elección cuando el LDX y el MPH no sean tolerados por el paciente. Por último, queremos incidir en la posibilidad que presentan los fármacos MPH, LDX y ATX de provocar efectos adversos a largo plazo, ya que son simpaticomiméticos y afectan al sistema cardiovascular. A corto plazo, no han demostrado ningún efecto secundario relevante. 


\section{Referencias bibliográficas}

Alda-Díez, José Ángel et al. 2017. Guía de Práctica Clínica sobre las Intervenciones Terapéuticas en el Trastorno por Déficit de Atención con Hiperactividad (TDAH). Recuperado de: https://portal.guiasalud.es/wpcontent/uploads/2018/12/GPC_574_TDAH_IACS_compl.pdf

Berg, Ansgar, Emelinn Bråtane, Hans Henrik Odland, Christina Brudvik, Bjørg Rosland y Asle Hirth. 2014. "Kardiovaskulær risikovurdering ved bruk av AD/HDmedikamenter hos barn». Tidsskr Nor Legeforen, 134: 710-714. doi: 10.4045/tidsskr.13.0565

Boerlin, Harold Louis, Michael J. Gitlin, Lori A. Zoellner y Constance L. Hammen. 1998. The Journal of clinical psychiatry. The Journal of Clinical Psychiatry (Vol. 59). [Physicians Postgraduate Press]. Recuperado de: https://www.psychiatrist.com/JCP/article/Pages/1998/v59n07/v59n0706.aspx

Dittmann, Ralf W., Esther Cardo, Peter Nagy, Colleen S. Anderson, Ralph Bloomfield, Beatriz Caballero, Nicholas Higgins, Paul Hodgkins, Andrew Lyne, Richard Civil y David Coghill. 2013. «Efficacy and safety of lisdexamfetamine dimesylate and atomoxetine in the treatment of attention-deficit/hyperactivity disorder: a headto-head, randomized, double-blind, phase IIlb study». CNS Drugs, 27(12): 1081-1092. https://doi.org/10.1007/s40263-013-0104-8

Hennissen, Leonie et al. 2017. «Cardiovascular Effects of Stimulant and Non-Stimulant Medication for Children and Adolescents with ADHD: A Systematic Review and Meta-Analysis of Trials of Methylphenidate, Amphetamines and Atomoxetine». CNS Drugs, 31(3): 199-215. https://doi.org/10.1007/s40263-017-0410-7

Karlstad, Øystein, Helga Zoëga, Kari Furu, Shahram Bahmanyar, Jaana E. Martikainen, Hellie Kieler y Anton Pottegård. 2016. "Use of drugs for ADHD among adults-a multinational study among 15.8 million adults in the Nordic countries». European Journal of Clinical Pharmacology, 72(2): 1504-1514. https://doi.org/10.1007/s00228-016-2125-y

King, Sarah, Susan Griffin, Zoé Hodges, Helen Weatherly, Christian Asseburg, Gerry Richardson, Su Golder, Eric Taylor, Mike Drummond y Robert Riemsma. 2006. «A systematic review and economic model of the effectiveness and costeffectiveness of methylphenidate, dexamfetamine and atomoxetine for the treatment of attention deficit hyperactivity disorder in children and adolescents". Health Technology Assessment, 10(23). https://doi.org/10.3310/hta10230

Linden, Stephan, Regina Bussing, Paul Kubilis, Tobias Gerhard, Richard Segal, Jonathan J. Shuster y Almut G. Winterstein. 2016. «Risk of Suicidal Events With Atomoxetine Compared to Stimulant Treatment: A Cohort Study». Pediatrics, 137(5): e20153199. https://doi.org/10.1542/peds.2015-3199

Newcorn, Jeffrey H., Peter Nagy, Ann C. Childress, Glen Frick, Brian Yan y Steven Pliszka. 2017. «Randomized, Double-Blind, Placebo-Controlled Acute Comparator Trials of Lisdexamfetamine and Extended-Release Methylphenidate in Adolescents With Attention-Deficit/Hyperactivity Disorder». CNS Drugs, 31(11): 999-1014. https://doi.org/10.1007/s40263-017-0468-2

Punja, Salima, Larissa Shamseer, Lisa Hartling, Liana Urichuk, Ben Vandermeer, Jane Nikles y Sunita Vohra. 2016. «Amphetamines for attention deficit hyperactivity disorder (ADHD) in children and adolescents». Cochrane Database of Systematic Reviews, 2(2): CD009996. https://doi.org/10.1002/14651858.CD009996.pub2

Soutollo-Esperón, César y Azucena Díez Suárez. 2007. Manual de diagnóstico y tratamiento del TDAH. Madrid: Médica Panamericana.

Storebø, Ole Jakob et al. 2015. «Methylphenidate for children and adolescents with attention deficit hyperactivity disorder (ADHD)». Cochrane Database of Systematic Reviews, 11(11): CD009885.

https://doi.org/10.1002/14651858.CD009885.pub2 
Vásquez, Josué, Eva Marcela Cárdenas, Miriam Feria, Corina Benjet, Lino Palacios y Francisco de la Peña. 2010. Guía clínica para el trastorno por déficit de atención e hiperactividad. México: Instituto Nacional de Psiquiatría Ramón de la Fuente Muñiz.

Zimovetz, Evelina A., Stephen M. Beard, Paul Hodgkins, Matthias Bischof, Josephine A. Mauskopf y Juliana Setyawan. 2016. «A Cost-Utility Analysis of Lisdexamfetamine Versus Atomoxetine in the Treatment of Children and Adolescents with Attention-Deficit/Hyperactivity Disorder and Inadequate Response to Methylphenidate». CNS Drugs, 30(10): 985-996. https://doi.org/10.1007/s40263-016-0354-3

Zimovetz, Evelina A., Alain Joseph, Rajeev Ayyagari y Josephine A. Mauskopf. 2018. «A cost-effectiveness analysis of lisdexamfetamine dimesylate in the treatment of adults with attention-deficit/hyperactivity disorder in the UK». The European Journal of Health Economics: HEPAC: Health Economics in Prevention and Care, 19(1): 21-35. https://doi.org/10.1007/s10198-016-0864-4 